\title{
2 Epitaxial growth of single-phase magnesium dihydride thin films
}

3 Ryota Shimizu*1,2 $^{* 1}$, Takuya Kakinokizono ${ }^{1}$, Igseon $\mathrm{Gu}^{1}$, and Taro Hitosugi ${ }^{1}$

$4{ }^{1}$ School of Materials and Chemical Technology, Tokyo Institute of Technology, 152-8552, Tokyo, 5 Japan

$6 \quad{ }^{2}$ PRESTO, Japan Science and Technology Agency, Kawaguchi, 332-0012, Japan 7

$8 \quad *$ corresponding author: shimizu.r.af@m.titech.ac.jp 
1 1. Optical transmittance measurements of $18 \mathrm{MgH}_{2}$ films fabricated under growth conditions in

3 Eighteen $\mathrm{MgH}_{2}$ films with the thickness of $60 \mathrm{~nm}$ were grown in accordance with conditions assigned

4 in the $L_{18}$ orthogonal array. The color of the films is shown in the photograph (Fig. S1(a)), and all the

5 optical transmittance $\left(T_{n}(n=1-18)\right)$ spectra of $18 \mathrm{MgH}_{2}$ sample in the wavelength of $200-900 \mathrm{~nm}$

6 are shown in Fig. S1(b), respectively. We found that the transmittance at wavelength of $400 \mathrm{~nm}$

7 strongly depended on the growth conditions.

(a)

\begin{tabular}{|c|c|c|}
\hline & ${ }^{\mathrm{H} 2} \mathrm{MgH}_{2}$ & ${ }^{\mathrm{n} 3} \mathrm{MgH}_{2}$ \\
\hline${ }^{\mathrm{Aa}} \mathrm{MgH}_{2}$ & ${ }^{\mathrm{A}} \mathrm{MgH}_{2}$ & ${ }^{\mathrm{H}} \mathrm{MgH}_{2}$ \\
\hline${ }^{47} \mathrm{MgH}_{2}$ & $\mathrm{MgH}_{2}$ & $\mathrm{MgH}_{2}$ \\
\hline$\stackrel{\mathrm{n} 10}{\mathrm{MgH}}{ }_{2}$ & ${ }^{\mathrm{H} 11} \mathrm{MgH}_{2}$ & ${ }^{\mathrm{H} 12} \mathrm{MgH}_{2}$ \\
\hline $\mathrm{MgH}_{2}$ & $\mathrm{MgH}_{2}$ & $\mathrm{MgH}_{2}$ \\
\hline${ }^{116}$ & $\mathrm{MgH}_{2}$ & ${ }^{\mathrm{H} 18} \mathrm{MgH}{ }_{2}$ \\
\hline
\end{tabular}

(b)

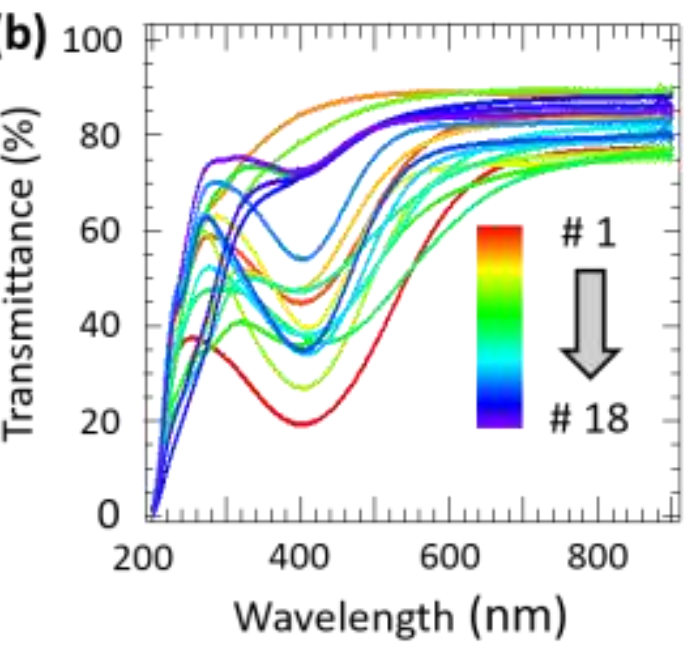

10 Figure S1: Results of $18 \mathrm{MgH}_{2}$ films fabricated in accordance with $L_{18}$ orthogonal array. (a)

11 Photographs of each $\mathrm{MgH}_{2} / \mathrm{MgO}(100)$ films. (b): Optical transmittance spectra of each

$12 \mathrm{MgH}_{2} / \mathrm{MgO}(100)$ film. 


\section{2. Calculation method for averaged responses in each condition}

2 We calculated the output response $\left(\eta_{n}=-\log _{10}\left(1 / T_{n}\right)^{2}(\mathrm{~dB})\right)$ in the $n$th $\mathrm{MgH}_{2}$ sample, and obtained each average response $\left(\eta_{\mathrm{ave}}(\mathrm{dB})\right)$ for each condition as follows:

4 Parameter 1: Degassing $\eta_{\text {ave }}($ Degassing: $\mathrm{N} / \mathrm{A})=\eta_{1}+\eta_{2}+\eta_{3}+\eta_{4}+\eta_{5}+\eta_{6}+\eta_{7}+\eta_{8}+\eta_{9}$

$\eta_{\text {ave }}($ Degassing: Degassed $)=\eta_{10}+\eta_{11}+\eta_{12}+\eta_{13}+\eta_{14}+\eta_{15}+\eta_{16}+\eta_{17}+\eta_{18}$

7 Parameter 2: $f_{\mathrm{H} 2}(\mathrm{sccm}): f_{\mathrm{Ar}}(\mathrm{sccm})$

$8 \quad \eta_{\text {ave }}\left(f_{\mathrm{H} 2} / f_{\mathrm{Ar}}:=5: 10\right)=\eta_{1}+\eta_{2}+\eta_{3}+\eta_{10}+\eta_{11}+\eta_{12}$

$9 \quad \eta_{\text {ave }}\left(f_{\mathrm{H} 2} / f_{\mathrm{Ar}}:=10: 10\right)=\eta_{4}+\eta_{5}+\eta_{6}+\eta_{13}+\eta_{14}+\eta_{15}$

$10 \quad \eta_{\text {ave }}\left(f_{\mathrm{H} 2} / f_{\mathrm{Ar}}:=15: 10\right)=\eta_{7}+\eta_{8}+\eta_{9}+\eta_{16}+\eta_{17}+\eta_{18}$

\section{Parameter 3: Pre-sputtering time (min.)}

$12 \eta_{\text {ave }}$ (Pre-sput. time: 2$)=\eta_{1}+\eta_{4}+\eta_{7}+\eta_{10}+\eta_{13}+\eta_{16}$

$13 \eta_{\text {ave }}$ (Pre-sput. time: 5$)=\eta_{2}+\eta_{5}+\eta_{8}+\eta_{11}+\eta_{14}+\eta_{17}$

$14 \quad \eta_{\text {ave }}$ (Pre-sput. time: 10$)=\eta_{3}+\eta_{6}+\eta_{9}+\eta_{12}+\eta_{15}+\eta_{18}$

\section{Parameter $4: \cdot$ Sample bias voltage}

$16 \eta_{\text {ave }}($ Sample bias: Floated $)=\eta_{1}+\eta_{4}+\eta_{9}+\eta_{11}+\eta_{15}+\eta_{17}$

$17 \quad \eta_{\text {ave }}($ Sample bias: Grounded $)=\eta_{2}+\eta_{5}+\eta_{7}+\eta_{12}+\eta_{13}+\eta_{15}$

$18 \eta_{\text {ave }}($ Sample bias: $-50 \mathrm{~V})=\eta_{3}+\eta_{6}+\eta_{8}+\eta_{10}+\eta_{14}+\eta_{16}$

\section{Parameter 5: Ar/H2 pressure (Pa)}

$20 \eta_{\text {ave }}\left(\operatorname{Ar} / \mathrm{H}_{2}\right.$ pressure: $\left.2 \times 10^{4}\right)=\eta_{1}+\eta_{6}+\eta_{7}+\eta_{11}+\eta_{14}+\eta_{18}$

$21 \eta_{\text {ave }}\left(\mathrm{Ar} / \mathrm{H}_{2}\right.$ pressure: $\left.5 \times 10^{4}\right)=\eta_{2}+\eta_{4}+\eta_{8}+\eta_{12}+\eta_{15}+\eta_{16}$

$22 \eta_{\text {ave }}\left(\mathrm{Ar} / \mathrm{H}_{2}\right.$ pressure: $\left.1 \times 10^{5}\right)=\eta_{3}+\eta_{5}+\eta_{9}+\eta_{10}+\eta_{13}+\eta_{17}$

\section{Parameter 6: Post-deposition annealing (PDA) temperature $\left({ }^{\circ} \mathrm{C}\right)$}

$24 \quad \eta_{\text {ave }}($ PDA temp.: 320$)=\eta_{1}+\eta_{6}+\eta_{8}+\eta_{12}+\eta_{13}+\eta_{17}$

$25 \eta_{\text {ave }}\left(\right.$ PDA temp.: 350) $=\eta_{2}+\eta_{4}+\eta_{9}+\eta_{10}+\eta_{14}+\eta_{18}$

$26 \eta_{\text {ave }}($ PDA temp.: 380$)=\eta_{3}+\eta_{5}+\eta_{7}+\eta_{11}+\eta_{15}+\eta_{16}$ 


\section{Parameter 7: Ramp rate $\left({ }^{\circ} \mathrm{C} / \mathrm{min}\right)$}

$2 \quad \eta_{\text {ave }}($ Ramp rate: 300$)=\eta_{1}+\eta_{5}+\eta_{9}+\eta_{12}+\eta_{14}+\eta_{16}$

$3 \quad \eta_{\text {ave }}($ Ramp rate: 100$)=\eta_{2}+\eta_{6}+\eta_{7}+\eta_{10}+\eta_{15}+\eta_{17}$

$4 \quad \eta_{\text {ave }}($ Ramp rate: 30$)=\eta_{3}+\eta_{4}+\eta_{8}+\eta_{11}+\eta_{13}+\eta_{18}$

\section{Parameter 8: Post-deposition annealing time (min.)}

$6 \quad \eta_{\text {ave }}($ PDA time: 3$)=\eta_{1}+\eta_{5}+\eta_{8}+\eta_{10}+\eta_{15}+\eta_{18}$

$7 \quad \eta_{\text {ave }}($ PDA time: 15$)=\eta_{2}+\eta_{6}+\eta_{9}+\eta_{11}+\eta_{13}+\eta_{16}$

$8 \eta_{\text {ave }}$ (PDA time: 30$)=\eta_{3}+\eta_{4}+\eta_{7}+\eta_{12}+\eta_{14}+\eta_{17}$

9 For optimization of growth conditions, we selected the set whose conditions yield the largest $\eta_{\text {ave }}$

10 values for each parameter. 


\section{3. Growth orientation control of $\mathrm{MgH}_{2}$ epitaxial thin films on $\mathrm{MgF}_{2}$ and $\mathrm{Al}_{2} \mathrm{O}_{3}$ substrate}

$\mathrm{MgH}_{2}$ (tetragonal (rutile), $a=b=4.517 \AA, c=3.021 \AA$ ) and $\mathrm{MgF}_{2}$ (tetragonal (rutile), $a=b=$

$44.620, c=3.051 \AA$ ) have the same rutile-type structure with similar lattice constants. Figure S2(a)

5 shows out-of-plane XRD patterns obtained in the films grown on $\mathrm{MgF}_{2}$ (100), (110), and (001)

6 substrates. To easily clarify the existence of epitaxial $\mathrm{MgH}_{2},(001)$-direction scans were also performed

7 for all the films grown on $\mathrm{MgF}_{2}(110),(100)$ and (001) substrates (Fig. S2(b)). All the samples show

$8 \mathrm{MgH}_{2} 002$ peak in the different $\psi$ angles, demonstrating rutile-on-rutile epitaxy.
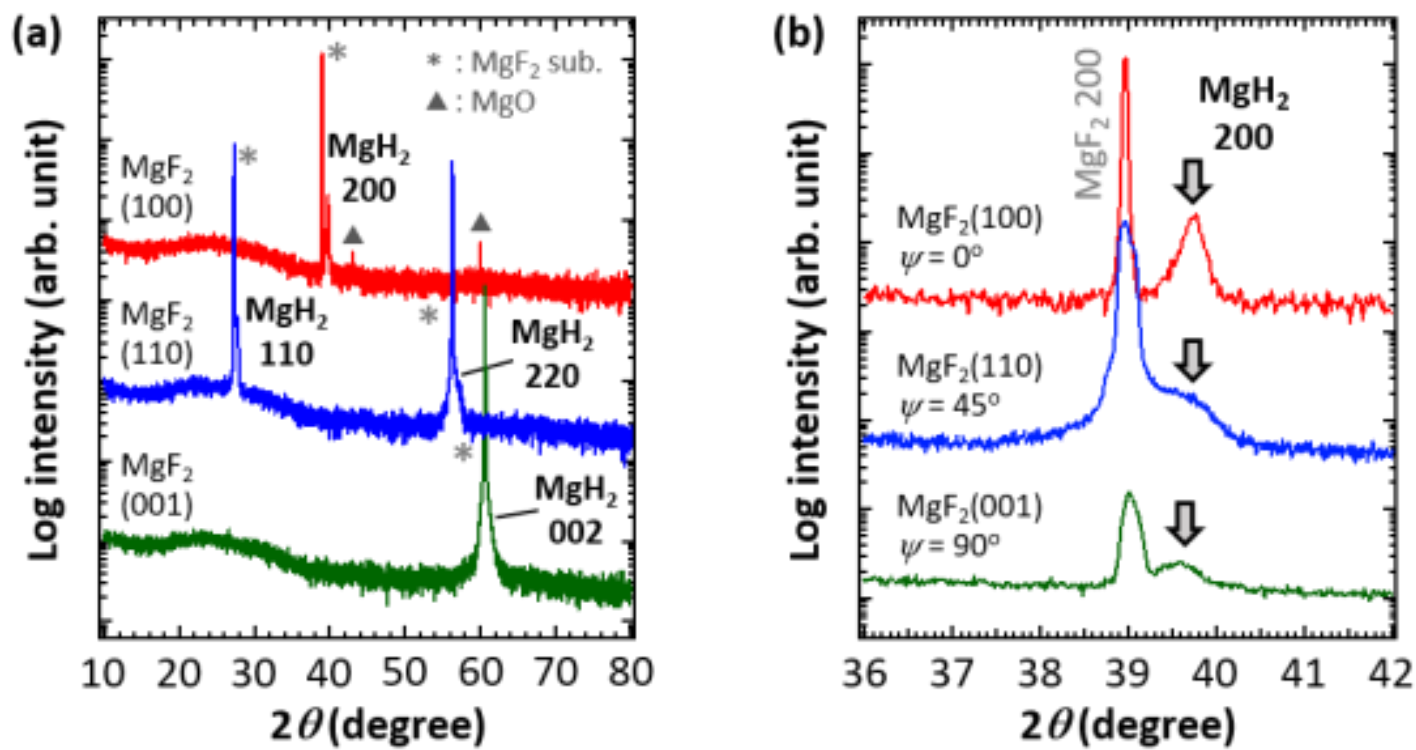

11 Figure S2: (a): Out-of-plane XRD patterns of $\mathrm{MgH}_{2}$ thin films grown on $\mathrm{MgF}_{2}$ (100, red), (110, blue),

12 and (001, green) substrates. (b): XRD patterns at around $\mathrm{MgF}_{2} 200$ spots. Bragg peaks of $\mathrm{MgH}_{2} 200$

13 were ideally observed in all the films. 
13.2 Epitaxial growth on $\mathrm{Al}_{2} \mathrm{O}_{3}(001)$ substrate

We also fabricated $\mathrm{MgH}_{2}$ epitaxial thin films on corundum-type $\mathrm{Al}_{2} \mathrm{O}_{3}(001)$ substrates. The out-

3 of-plane scan and pole figure measurements demonstrate (100)-oriented epitaxial growth, in a similar 4 way to rutile- $\mathrm{TiO}_{2}$ on $\mathrm{Al}_{2} \mathrm{O}_{3}(001)$ substrates [1].

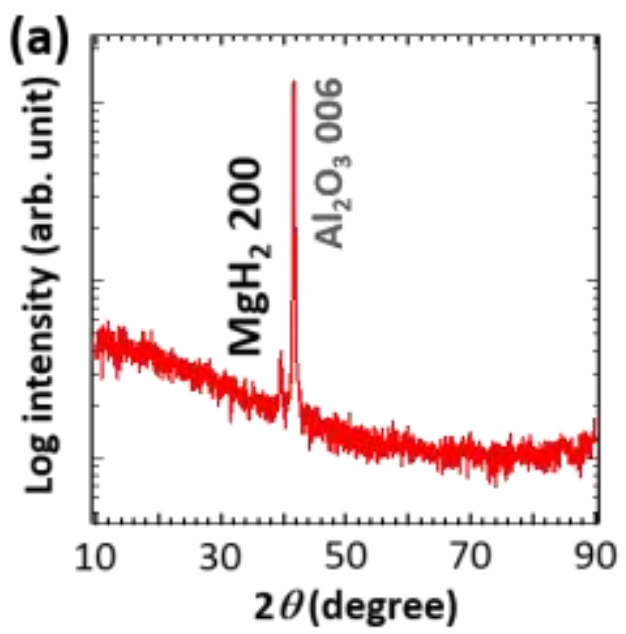

(b): $\mathrm{MgH}_{2} 110$ (c): $\mathrm{Al}_{2} \mathrm{O}_{3} 119$

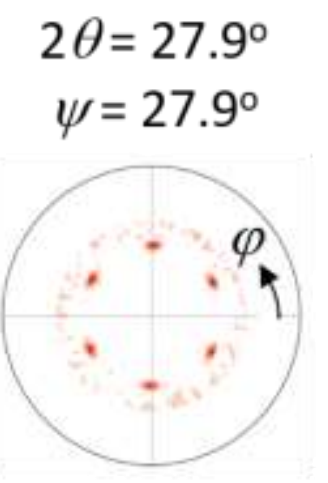

$$
2 \theta=77.2^{\circ}
$$$$
\psi=31.2^{\circ}
$$

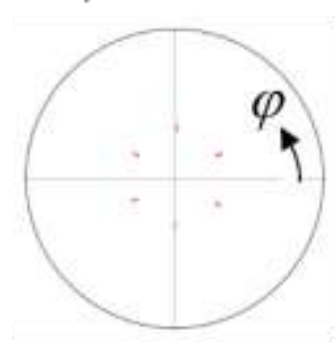

6

\section{Reference}

13 [1]: Chen, S., Mason, M. G., Gysling, H. J., Paz-Pujalt, G. R., Blanton, T. N., Castro, T., Chen, K. M., 14 Fictorie, C. P., Gladfelter, W. L., Franciosi, A., Cohen, P. I., and Evans, J. F. "Ultrahigh vacuum 15 metalorganic chemical vapor deposition growth and in situ characterization of epitaxial $\mathrm{TiO}_{2}$ films", $\mathrm{J}$. 16 Vac. Sci. Technol. A11, 2419 (1993). 